\title{
La literatura como búsqueda. Conversaciones con Rafael Chirbes
}

Serber, Daniela Cecilia

Universidad del Salvador, Argentina

Cita sugerida: Serber, D.(2018). La literatura como búsqueda. Conversaciones con Rafael Chirbes. En C. Somolinos Molina (ed.), "Escrituras del cuerpo: Marta Sanz". Olivar, 18 (27), e027. https://doi.org/10.24215/18524478e027

(c) (1) (2) (2) Esta obra está bajo licencia Creative Commons Atribución-NoComercial-CompartirIgual 4.0 Internacional http://creativecommons.org/licenses/by-nc-sa/4.0/deed.es_AR 


\title{
La literatura como búsqueda. Conversaciones con Rafael Chirbes
}

\author{
Daniela Cecilia Serber \\ Universidad del Salvador, Argentina
}

El 15 de agosto de 2018 se cumplirán tres años del fallecimiento del escritor valenciano Rafael Chirbes y dos de la apertura de la fundación que lleva su nombre, albergada por su casa de Beniarbeig, Alicante, concreción de uno de los deseos expresos que dejó en manos de familiares y amigos. Para ese entonces, se habrá realizado ya el I Congreso Internacional “El Universo de Rafael Chirbes” —organizado por la fundación y la Universidad de Valencia-, que se llevará a cabo entre el 9 y el 12 de mayo, a treinta años de la aparición de Mimoun (1988), primera nouvelle publicada del autor.

Son sus sobrinos, María José y Manuel Micó, los que encabezan la Fundación Rafael Chirbes, uno de cuyos objetivos, como se explica en su página web oficial, es fomentar el estudio y la difusión de su obra, como así también custodiar su legado literario. El mismo Manuel, en nuestro primer contacto electrónico personal, me explicaba que están recopilando trabajos de investigación y, asimismo, intercambios epistolares que podrían transformarse en una muestra para estudiar al autor y su obra. En este caso, continuaba, decidieron no leer sus correos, sino poner en marcha el proceso contrario: solicitarles a sus interlocutores que aportaran la correspondencia.

Es en este contexto que se presentan a continuación — previa consulta a sus sobrinos - fragmentos de la comunicación electrónica que mantuve con don Rafael entre julio de 2013 y primeros meses de 2015, a veces esporádica por diversos motivos, pero siempre valiosa. Este intercambio nació como una entrevista académica, formal y estructurada sobre temas específicos, con visos teóricos, cuyas preguntas habían surgido en el fragor de la lectura de la obra chirbesiana y de textos teóricos y críticos para la elaboración de una monografía de posgrado en la Universidad Nacional de Educación a Distancia (UNED), España, sobre la nouvelle Los disparos del cazador (1994). Esa monografía daría origen a mi trabajo de fin de máster (TFM) que gira en torno de la (re)lectura y (re)escritura de la Historia española reciente que Rafael Chirbes propone desde la literatura. Allí analizaba Mimoun y Los disparos del cazador, pero también La caída de Madrid (2000) y En la orilla (2013), que era, en ese momento, su más reciente obra.

Pero el rígido cuestionario que tímidamente le envió la alumna que yo era entonces, en pleno descubrimiento de todo su mundo novelesco, se transformó en un cálido ida y vuelta gracias a la generosidad y la sencillez de don Rafael. Por eso, este texto es, además de la reunión de sucesivos intercambios entre una lectora y su estudiado y admirado escritor, una semblanza de Rafael Chirbes a modo de homenaje.

\section{EL PRIMER CONTACTO}

Le solicité la entrevista el 8 de mayo de 2013: "Me interesan mucho los temas que usted aborda en sus textos -le decía-, pero, principalmente, la forma en que lo hace, el ángulo desde el cual nos invita a los lectores a reflexionar. Cuando hoy la metaliteratura ha ganado terreno, a mí me cautiva el relato y disfruto mucho de 
sus historias". La primera de todas las amables respuestas de don Rafael llegó solo tres días después, el 11 de mayo; se ponía a disposición y cerraba su correo con un humilde saludo, que (lo sabría más adelante) era un sello de su personalidad, como lo confirman sus amigos y entrevistadores en más de una ocasión: "No quiero despedirme sin darle las gracias por su atención a mis libros. Un saludo afectuoso. Rafael”.

Chirbes estaría de viaje durante mayo y junio, por lo que le envié las preguntas en julio después de otras tantas lecturas. Ese primer cuestionario, cargado de reflexiones teóricas, fueron contestadas el 23 de agosto del modo menos esperado, pero aleccionador: nuestro escritor respondía con el prólogo a la edición aniversario de La buena letra y Los disparos del cazador, titulada Pecados originales (2013), aún inédito para ese entonces.

Estimada Daniela:

Disculpe mi tardanza, me acerco poco por el correo de internet. A lo largo del día de hoy procuraré responderle, aunque me animo a enviarle el prólogo que he hecho para la nueva edición —esta vez conjunta- de Los disparos y La buena letra; quizá su lectura le aclare buena parte de las cosas que me pregunta. Le ruego que me lo diga para así ajustar mis respuestas.

Un saludo afectuoso.

Rafael

Ese prólogo era, efectivamente, de gran valor para mí y, como le expresé en mi inmediato agradecimiento, resolvía algunas de mis inquietudes e iluminaba el trabajo que redactaba por entonces. Sin embargo, intenté que se refiriera a aspectos puntuales de mi cuestionario: algunas de esas respuestas llegarían más adelante, al año siguiente, y otras nunca llegarían. En ese camino, entendí que don Rafael escapaba de las teorizaciones y se rehusaba a explicar sus libros, como lo planteó - entre otras ocasiones- en varias entrevistas ${ }^{1}$.

Por ello, desarmé la madeja de preguntas iniciales y fui solo proponiendo algunos de esos hilos en una conversación — por momentos, extensa y, por otros, más breve-, que, en ocasiones, se desviaba de su primer propósito. Así culminó 2013.

\section{RECORRIENDO EL MUNDO CHIRBESIANO}

Recién el 6 de mayo del año siguiente, volví a contactarme. Le contaba ya mi trabajo sobre el aspecto de la (re)lectura y (re)escritura de la Historia en algunas de sus obras: por entonces, En la orilla me tenía atrapada y preparaba dos comunicaciones académicas. Una de ellas, abordaba “Textos ventaneros (Diario)", publicados en $E$ ñe. Revista para leer (Chirbes, 2009), presentados como fragmentos del diario del autor y que me resultaron interesantes e iluminadores por varios motivos. De allí, mi consulta sobre esos escritos y su contexto. Doce días después, su respuesta fue concisa, pero me confirmaba lo que ya encontraba como un tema recurrente en su obra:

\section{Daniela Serber:}

- ¿Podría contarme la importancia de la librería La Fábrica de Madrid y la revista Eñe 2 ?

- ¿Cuál fue la propuesta concreta de la librería? ¿Le dio indicaciones, estableció condiciones sobre la escritura? (sobre temas, sobre el carácter veridico of ficticio del texto, sobre la extensión).

- ¿Quélo motivó a responder a la propuesta?

- ¿Fue un texto integramente escrito para esa ocasión o realmente lleva un diario y el texto es una parte de él? Hay una referencia concreta al pedido en el texto.

\section{Rafael Chirbes:}

Estimada Daniela:

Le ruego que me perdone mi tardanza en responderle, estoy bastante distraído y apenas me acerco a internet. No tengo ninguna relación con La Fábrica, me pidieron unas páginas de diario y las tomé de unos cuadernitos que, de vez en cuando, llevo, nada con voluntad de publicación, más bien una manera de poner orden en mi cabeza.

Es la escritura como instrumento ordenador ese tema recurrente: como él mismo explica en "Textos ventaneros (Diario)" y también en algunas entrevistas — recordando a su amiga y mentora Carmen Martín Gaite-, la escritura es imprescindible para "fijar, dejar constancia, hacer que las cosas hayan ocurrido [...] 
cazar, capturar, ordenar las ideas para que tengan existencia [...]" (Chirbes, 2009) ${ }^{3}$. Muchos de sus personajes se preocupan y hasta se obsesionan por ello, por legar su versión de la historia, aquella que entra en conflicto con los grandes discursos oficiales, la que pugna por no caer en el olvido.

En ese mismo correo electrónico del 6 de mayo, le preguntaba, justamente, sobre el valor del testimonio y sobre la función de la literatura en este sentido: empezaba a vislumbrar la importancia en su obra de esas voces individuales, de la figura del testigo, y también la convicción de don Rafael de que la literatura debe intervenir en la realidad.

D.C.: ¿Quépapel cumplen las visiones subjetivas/personales en la (re)construcción de la historia y la memoria? ¿Quépapel ocupao debería ocupar para usted la literatura en la construcción de esa historia y esa memoria? ¿Es esto visible en la actualidad española? R.C.: Creo que la literatura es parte indispensable para la construcción de eso que ahora llaman "el relato" de una época: las distintas áreas de la cultura son las que modelan la sensibilidad y las aspiraciones de la gente, y todas luchan aunque no lo sepan por imponer su propia narración, su idea de la historia y de las cosas.

Gracias por su generosa lectura de mis libros, no tenga ningún reparo en ponerse en contacto conmigo cuando lo desee.

Prometo ser más rápido en la respuesta.

Un saludo afectuoso.

Rafael

A pesar de su invitación, siempre temí transformarme en una seguidora indeseable y hoy pienso que, por ello, perdí algunas oportunidades de enriquecerme con sus correos. Me contacté unos meses después, en septiembre, con muchas más lecturas de crítica de su obra y también entrevistas. Pero, fundamentalmente, había empezado a trabajar con En la orilla: "me dejó llena de preguntas, sin aire por momentos. Plantea tantas cuestiones históricas y existenciales... Ancla en España y su historia, pero se proyecta a un proceso global y también al más íntimo e individual del ser humano. Me emocionó y me hizo reflexionar, ambas cosas". Y compartí con él que presentaría un avance de las ideas de mi análisis en el III Congreso Internacional de Literatura y Cultura Españolas Contemporánea en la Universidad Nacional de La Plata, que se desarrolló en octubre de 2014. Le contaba que, en congresos argentinos, se exponían trabajos sobre sus obras y que se incluían en los programas de algunas universidades. Mi casa de estudios no era (ni es aún hoy) la excepción y le comenté que, además, transitábamos la lectura de escritores del exilio republicano español, como León Felipe y Max Aub, un referente fundamental para Rafael Chirbes. Le transmití la experiencia con mis alumnos y su fascinación, como así también la línea que establecíamos entre esas lecturas y su novelística.

En ese contexto, le envié cinco preguntas para seguir reflexionando sobre su obra, que, días después, fue contestando entre mis líneas:

D.C.: En El novelista perplejo y en varias entrevistas, usted hace referencia de forma recurrente a escritores españoles que admira, como Pérez Galdós, Max Aub o la generación del 50. ¿Qué ha influido especificamente en su escritura de cada uno de ellos y desde qué aspecto?

R.C.: Creo que, en los tres casos que me propone, busco que la literatura sea una búsqueda por entender los mecanismos del mundo que me rodea y mi lugar en él. La literatura como búsqueda y el concepto de belleza ligado al de conocimiento, es bello lo que me hace ver las cosas - las que sean- desde un lugar nuevo y más rico. Saber que el estilo, siendo lo único (un libro es escritura), es fruto de ese esfuerzo y, como diría el evangelio, se nos dará por añadidura. Carmen Martín Gaite decía que la urgencia trae la forma.

D.C.: ¿Se siente influido, identificado, en diálogo, con alguno de sus colegas en la actualidad?

R.C.: Me interesa mucho esa escritura salvaje de Miguel Sánchez-Ostiz. Envidio su energía, su rabia.

D.C.: En "Las razones de un libro", que encabeza El novelista perplejo, comenta que, en algunos momentos de su obra, ha abordado los porqués intimos de su escritura "de refilón, a través de personajes que no eran yo, o que sólo de refilón eran yo, o que eran tan yo que parecian otros"; y, en "El punto de vista", que "un novelista nos entrega, con la radiografia de su tiempo, su propia radiografia". ¿Qué tienen de usted los personajes de sus novelas, aun aquellos que se encuentran en una vereda ideológica opuesta a la suya?

R.C.: Todos son yo.

D.C.: La focalización de la historia desde personajes franquistas o complacientes con el régimen y la hipocresía de sus hijos, que usted identifica con su generación... ¿Cómo ha sido recibido esto por quienes son o fueron sus compañeros ideológicos? ¿Y cómo se ha recibido en el contexto de los movimientos de recuperación de la memoria histórica? 
R.C.: No sabría decirle. Al principio, con cierta indiferencia; ahora, con un extraño acuerdo general

D.C.: ¿Cómo esperaba que se leyera el presente de España a partir de Mimoun y Los disparos del cazador en el momento de supublicación? ¿Qué espera que transmitan hoy? ¿Cómo espera que se lea En la orilla?

R.C.: Quise que se leyera lavada de toda esa baba retórica que generó la transición con sus héroes de papel prensa.

En pocas líneas, algunos de los principios chirbesianos: la literatura como búsqueda, el punto de vista como elemento fundamental, la preocupación por el contenido y su voluntad de desmitificar la Historia. Asimismo, una elocuente síntesis de la trayectoria de sus libros en España - de "cierta indiferencia" a "un extraño acuerdo general" - que deja en evidencia que siempre se reservaba el derecho a dudar del éxito.

\section{ÚLTIMOS CONTACTOS}

En febrero de 2015, le envié, una vez más, un extenso correo electrónico con preguntas variadas que seguían surgiendo en el proceso de lectura y escritura ${ }^{4}$. Estaba abocada al capítulo sobre Mimoun, en el que retomaba la idea - planteada en una tesis de doctorado- de que se trata de una "novela prólogo" de su proyecto narrativo (Muñoz, 2009) e intentaba profundizar en la lectura política de esa primera nouvelle, que Alemania - país en el que Chirbes tuvo éxito desde el primer momento- hizo inmediatamente y que, en España, llegó más tarde, tal como él mismo señalaba en más de una ocasión:

D.C.: En la entrevista otorgada al periódico argentino La Nación (Serrano, 2014), se menciona que una de sus obras fue adaptada al teatro. ¿Es correcto esto? En ese caso, ¿cuál fue la obra adaptada?

R.C.: No hay ninguna novela mía adaptada al teatro, hay una productora que está en ello, y al parecer la estrenará a finales del 2015.

[Hoy sabemos que esa obra es En la orilla, adaptada y dirigida por Adolfo Fernández, y estrenada en Alicante el 3 de marzo de 2017. Es una coproducción que sigue en cartel y está de gira por España. Tuvo, desde su estreno, un gran éxito, como lo demuestran diversas reseñas y comentarios. En algunos artículos de diario recientes, ya se anuncia su transposición fílmica].

D.C.: En una entrevista en la Universidad de Cádiz (en el ciclo Literarias), usted comentó que la lectura politica de una novela es absurda, porque la novela es otra cosa (Heredia, 2010). ¿Podría desarrollar esta idea?

R.C.: Si dije eso de literatura y política en la Universidad de Cádiz, o me expresé mal o lo captó mal el periodista. La literatura, incluida la novela, es ideología, muestra una idea del mundo, y como tal participa en la lucha de los imaginarios que pugnan por el poder. Pero también creo que la novela trabaja en un nivel distinto de la sociología, el periodismo de denuncia o los avatares y consignas inmediatos de los partidos políticos, tiene otro paso, aspira a mirar desde otro espacio en la organización de un texto que sea capaz de revelar aspectos que nadie más puede revelar. Si pensamos que no está capacitada para eso, para ocupar un lugar insustituible, entonces es que la damos por muerta. Retórica.

D.C.: En varias entrevistas, usted comenta cuánto influyó su formación en Historia en sus novelas. En relación con esta cuestión, le pregunto lo siguiente:

- En un artículo del último número de la revista Turia ${ }^{5}$, usted comenta: "La historia es una carniceria". ¿A qué se refiere exactamente?

- López Abiada, en otro artículo de la misma revista, menciona que, en una entrevista con Wolfgang Höbel ante la publicación de En la orilla, se definió como "historiador" ("Deseo ser veraz. Soy historiador"). ¿Cómo se relacionan el historiador y el novelista en el momento de la escritura?

R.C.:

a) Lo de la historia es carnicería va en el sentido de las lecciones de filosofía de la historia de Benjamin, tan citadas. Los vencedores se apropian del botín.

b) No entiendo la novela sin historia, no entiendo nada sin historia, es que no lo hay; todo tiene su tiempo y es de su tiempo.

En mayo de ese mismo año 2015, un nuevo intercambio. No podía imaginar que sería el último. En mi correo, le contaba el final de la escritura del TFM y recordaba que se cumplían dos años de nuestro primer contacto. Su última respuesta tardó solo un día en llegar. Hoy, a la luz de la tarea de la Fundación Rafael Chirbes, del inminente I Congreso Internacional sobre su obra, de la publicación de El año que nevó en Valencia (2017) en la reeditada colección Nuevos Cuadernos Anagrama —uno de esos cuentos cuya 
existencia, por entonces, yo no conocía $^{6}$ - y la traducción al valenciano de La buena letra $^{7}$, algunas de sus respuestas se llenan de nuevos significados.

En ese mensaje, le preguntaba sobre sus cuentos y le pedía "pistas" para rastrearlos todos:

D.C.: Continué profundizando en su obra y sigo encontrando nuevas aristas; siempre me deja nuevas preguntas y suscita reflexiones. Me gustaria leer sus cuentos también. En relación con ellos, una pregunta: lei que publicó ocho cuentos. De momento, encontré la referencia de dos: "Temporada baja" (publicado en Revista de Occidente) y "Un cuento de invierno" (publicado en la revista BITZOC Literatura). Si no es mucha molestia, ¿podría saber el titulo de los otros para poder buscarlos?

R.C.: No he escrito cuentos, tres o cuatro que andan por ahí perdidos y no merecen sus molestias.

Me atrevo a contradecirlo y pienso que sí merecen "la molestia": creo que la reedición de El año que nevó en Valencia lo confirma.

Le pregunté, asimismo, sobre la lengua que, siendo valenciano, había elegido para su escritura: el castellano. Su respuesta fue, quizás, una de las más contundentes ya que, con pocas palabras, transmite la dimensión de lo que significó el franquismo en la vida cotidiana de los ciudadanos de a pie, que encarnan la intrahistoria de los pueblos:

D.C.: Otra pregunta que me surgió al leer sus entrevistas y la biocronologia que Fernando del Val publicó en la revista Turia N ${ }^{\circ} 112^{8}$, cuyo "Cartapacio" le dedicaron, es si escribió algún texto en valenciano o siempre lo hizo en castellano.

R.C.: Nunca he escrito en valenciano; a pesar de que es mi lengua materna, soy un analfabeto hijo del franquismo. La leo, pero me da vergüenza escribirla porque cometo faltas de ortografía; además - y sobre todo - como he vivido casi toda mi vida en zonas castellanoparlantes, es una lengua en la que me expreso como un niño de Tavernes, que es donde nací y aprendí a hablar.

Hoy, La bona lletra, es la primera traducción que, de alguna manera, intenta suturar esa herida de la Historia.

Por último, en mi correo, comentarios y preguntas sobre La caída de Madrid —novela que se desarrolla en un solo día, la víspera de la muerte de Francisco Franco, pero que abarca la historia española desde los años 40 hasta los $80-$ y otra respuesta contundente:

D.C.: Me puse a pensar lo que deben de haber sentido sus lectores españoles: la revisión de esos 25 años que separan la historia de estos personajes y la publicación de la novela [en 2000], todo lo que quedó en el camino. Me recordó, en este sentido, a La de Bringas, de Pérez Galdós [otro de los referentes de Rafael Chirbes]: la desilusión de ese hombre tras 16 años de la Gloriosa.

Me preguntaba si la situación del profesor Chacón [personaje exiliado, sobreviviente de los campos de concentración del sur de Francia, jubilado ya de su puesto de profesor de literatura en la Universidad Autónoma de México, que retorna a España, pero elige el silencio] está aún vigente o si su "constelación", como diría Benjamin, ha encontrado un lugar ${ }^{9}$. Quiero decir, si en España los exiliados y su obra se han ido reincorporando en "el relato", como usted dice. Sé que hay muchas deudas aún desde el punto de vista politico. ¿Pero quépasa con el pueblo, con los lectores? En virtud de ello también me preguntaba, entonces, si el profesor Chacón hoy seguiria pensando lo mismo, si su sensación seria la misma. ¿Qué diría hoy a los españoles?

R.C.: La literatura del exilio dormita, fue expulsada en la transición.

Y me recomendó la lectura de un libro que, decía,

R.C.: [...] sostiene la tesis de que cuando llegaron a España los exiliados ya no le interesaban a nadie. Creo que debería usted leer ese libro [...] para entender la tesis que se ha querido imponer desde la casta dominante interesada en no remover un pasado que muestra la radical ilegitimidad de la actual democracia española ${ }^{10}$.

Ese fue su último correo: “Gracias por la atención con que lee mis libros”, decía una vez más. Yo continué escribiendo hasta que volé a Madrid, en junio de 2015: nunca sabré si esos mensajes fueron leídos. En ellos, extensos, más preguntas y los datos de mi viaje — así, como al pasar-, esperando que me confirmara alguna charla o presentación que siguiera a las de la Feria del Libro de Madrid y a la de un festival en Girona, con la secreta ilusión de encontrarlo en un rincón de España. No pudo ser. A pocos días de mi regreso a Buenos Aires, entendí, de un golpe, su silencio. Nos quedan sus libros para seguir conversando con él y continuar la búsqueda. 


\section{REFERENCIAS}

Barjau, Teresa y Joaquim Parellada, 2013. "Rafael Chirbes, en Beniarbeig”, Insula, 803, 13-21.

Chirbes, Rafael, 1988. Mimoun, Barcelona: Anagrama/Narrativas hispánicas.

Chirbes, Rafael, 2000. La caída de Madrid. Barcelona: Anagrama/Narrativas hispánicas.

Chirbes, Rafael, 2003 [1994]. Los disparos del cazador. Barcelona: Anagrama/Compactos.

Chirbes, Rafael, 2009. “Textos ventaneros (Diario)", Eñe. Revista para leer. Recuperado de http:// www.elboomeran.com/upload/ficheros/noticias/diariochirbes.pdf

Chirbes, Rafael, 2013a. En la orilla. Barcelona: Anagrama/Narrativas hispánicas.

Chirbes, Rafael, 2013b. "Un escritor egoísta”. En Pecados originales: La buena letra \& Los disparos del cazador. Barcelona: Anagrama/Otra vuelta de tuerca.

Heredia, Daniel, 2010. Literarias: Rafael Chirbes. Universidad de Cádiz. Recuperado de http://www.youtube.com/ watch?v=1bOdnT-qhM8 (parte 1) http://www.youtube.com/watch?v=tBJZWG41V4M (parte 2) http:// www.youtube.com/watch?v=wycSlvRmxV0 (parte 3) http://www.youtube.com/watch?v=vqVX3kWrrWo (parte 4)

Muñoz, Ignacio, 2009. "Capítulo IV. Memoria crítica de la Transición en la narrativa de Rafael Chirbes", en $L a$ reivindicación de la memoria colectiva en la narrativa española contemporánea (1986-2006): Ramiro Pinilla, Rafael Chirbes y Manuel Longares. Tesis doctoral dirigida por Francisco Caudet, Universidad Autónoma de Madrid, Facultad de Filosofía y Letras, Departamento de Filología Española. Mimeo.

Serrano, Violeta, 2014. "En mi trabajo literario procuro poner cuanto sé", La Nación. Recuperado de http:// www.lanacion.com.ar/1745535-en-mi-trabajo-literario-procuro-poner-cuanto-se

Val, Fernando del, 2014-2015. "Biocronología de Rafael Chirbes", Turia. Revista cultural, 112, 280-305.

\section{Notas}

1 Cfr., por ejemplo, Barjau y Parellada, 2013: 13-21.

2 Eñe. Revista para leer es editada desde 2005 por la librería La Fábrica de Madrid (http://www.lafabrica.com) y está dedicada a la literatura contemporánea. En el momento del intercambio con Rafael Chirbes, era una publicación en soporte papel y con sitio web propio en el cual permitía el acceso libre y gratuito al índice y a algunos artículos o secciones. La página web ha crecido (http://revistaparaleer.com/) y, actualmente, se puede adquirir la revista en soporte papel o en versión digital en varias plataformas en línea.

3 Archivo digital sin paginación.

4 Dos preguntas quedaron sin responder:

- Cuando recibió el Premio de Narrativa por En la orilla, aseguró que, si tuviera la oportunidad, le diría al ministro Wert lo que piensa de su gestión. ¿Cuáles son sus principales críticas?

- En una entrevista en 2007 a Nuria Anzacot de El Cultural, tras la publicación de Crematorio,se aludía a un próximo proyecto: un libro sobre el trabajo, cuyo modelo sería Gran Sol de Aldecoa. La obra que conocimos después es En la orilla. ¿Fue esa la idea germen de la novela o se refería a algún otro texto que no vio la luz?

5 Turia. Revista cultural, número 112, noviembre 2014-febrero 2015.

6 Fue publicado por primera vez en Cuadernos de la Mangana en 2003.

7 Presentada en febrero de este año. La obra fue traducida por Carles Mulet, prologada por Alfons Cervera y editada por Letra Impresa Ediciones, en colaboración con la Fundación Rafael Chirbes.

8 Val, 2014-2015: 280-305.

9 La crítica suele asociar al profesor Chacón con Max Aub por los datos de su vida y por su desazón tras esa vuelta, tan alejada de sus expectativas. A diferencia de Aub, sin embargo, el personaje chirbesiano, como dijimos, se silencia.

10 Considero prudente, en este caso, reservar el título del libro y el nombre del autor. Además, lo esencial de estas líneas es que reflejan la mirada crítica de Chirbes respecto de esa tesis y su postura en el debate, aún actual, en torno a la democracia española. 